\title{
Características químicas e sensoriais de mortadelas produzidas com diferentes proporções de carne de frango mecanicamente separada
}

\author{
Sensory and chemical characteristics of bologna produced with \\ mechanically deboned chicken meat
}

Mônica Queiroz de Freitas, ${ }^{\star}$ José Benício Paes Chaves, ${ }^{\star \star}$ Valéria Paula Rodrigues Minim, ${ }^{\star \star}$ Lúcio Alberto Miranda Gomide ${ }^{\star \star}$

\begin{abstract}
Resumo
O experimento foi delineado para demonstrar o efeito da adição de Carne Mecanicamente Separada (CMS) sobre as características sensoriais e físico-químicas de embutido cozido. Para tanto, foram processadas 11 formulações de mortadelas, cujas porções cárneas variaram entre 0 e $100 \%$ de CMS de frango, em substituição à carrie de coxa e sobrecoxa de frango desossada manualmente. Para a análise sensorial descritiva utilizou-se o método de Análise Descritiva Quantitativa (ADQ), com equipe de oito julgadores, os quais avaliaram atributos de aparência, aroma, sabor e textura das mortadelas. De modo geral, os resultados obtidos na composição centesimal se apresentaram dentro dos padrões estabelecidos para o produto, e a adição de CMS reduziu a elasticidade, a coesividade e a dureza das mortadelas. Nas mortadelas de frango estudadas, ficou claramente demonstrado que a adição de CMS contribuiu para acentuar a cor, o aroma e o sabor de CMS, e o aroma e sabor característico, aproximando-as das mortadelas tradicionais, produzidas com carne de diversos animais de açougue.
\end{abstract}

Palavras-chave: características químicas e sensoriais, mortadelas, frango.

\begin{abstract}
This research was designed to demonstrate the effects of adding mechanically deboned chicken (MDC) meat on some chemical and sensory characteristics of cooked bologna. Eleven formulations of bologna were prepared, in which, the meat portion ranged from zero to $100 \%$ MDC meat, in substitution to chicken tight and leg manually deboned. Eight trained judge evaluated 16 sensory attributes related to appearance, aroma, flavor and texture of the bolognas. The results obtained for the gross chemical composition were well within the normal range for this type of product, meeting regular Brazilian official standards. The addition of MDC meat to the bolognas reduced its sensory elasticity, cohesiveness and hardness. Also, bolognas with higher levels of MDC meat had their color, aroma and flavor closer to that of MDC. Furthermore, addition of MDC meat improved the characteristic aroma and flavor of traditionally produced bolognas.
\end{abstract}

Keywords: sensory chemical characteristics, bologna chicken.

\section{Introdução}

O aumento das exportações e do consumo interno de frango em cortes tem aumentado a produção de Carne Mecanicamente Separada (CMS) de frango e incrementado a sua adição em diversos produtos cárneos cozidos, em substituição à carne de frango desossada manualmente.

O processo de separação mecânica ocasiona ruptura das miofibrilas da carne (Schnell et al., 1974), desnaturações protéicas, aumento do conteúdo de hemopigmentos (Froning, 1981) e de resíduos ósseos (Field, 1974), além de incorporar lipídeos, provenientes, principalmente, da medula óssea e da pele de frango (Moerk e Ball, 1974 e Lee et al. , 1975). A composição centesimal, aproximada, da CMS de frango é de
62 a $70 \%$ de umidade, 14 a $23 \%$ de gordura e 12 a $17 \%$ de proteina (Humm e Young, 1983).

Segundo Shand et al. (1990), as propriedades funcionais dos sistemas cárneos dependem das interações proteína-água, proteina gordura e proteína-proteína, as quais refletem diretamente nas características sensoriais dos alimentos. Neste sentido McMahon e Dawson (1976) verificaram que a substituição da carne desossada manualmente, pela CMS, prejudicava a estabilidade da emulsão em virtude de sua menor capacidade emulsificante. Mais tarde, McMilin et al. (1980) e Meullenet et al. (1994) estudaram o efeito da CMS sobre as características de textura de salsichas e observaram que dureza, coesividade, elasticidade, mastigabilidade e suculência foram os atributos mais expressivos na descrição

\footnotetext{
* Professora da Faculdade de Medicina Veterinária da Universidade Federal Fluminense, Rua Vital Brazil Filho, 64. CEP 24230-340 - Niterói, RJ - Brasil

** Professores do Departamento de Tecnologia de Alimentos da Universidade Federal de Viçosa, MG - 36571-000 - Viçosa, MG
} 
do produto. Segundo Froning (1976) e Raphaelides et al. (1998), a quantidade três vezes maior de hemopigmentos, além de aumentar o conteúdo lipídico, torna a CMS mais vermelha do que a carne de frango proveniente da desossa manual. Segundo Dawson e Gartner (1983), os fragmentos ósseos presentes na CMS conferem características sensoriais geométricas de textura, aos alimentos que a contenham, descritas como arenosa e granulosa.

Diante do exposto, o presente trabalho teve por objetivo analisar a composição centesimal, os atributos sensoriais de aparência, aroma, sabor e textura empregados na descrição de mortadelas de frango formuladas com diferentes proporções de CMS, em substituição à carne de frango desossada manualmente, como também, a relação funcional dos níveis de CMS com tais atributos.

\section{Material e métodos}

Uma parte da carne de frango utilizada na produção das mortadelas foi proveniente da desossa manual de coxa e sobrecoxa e outra proveniente da separação mecânica de dorso $(20 \%)$ e pescoço $(80 \%)$, sem a pele. Foram produzidas mortadelas com massa básica constituída, em média, de $60 \%$ de carne de frango, 3 a $6 \%$ de proteína de soja, 5 a $20 \%$ de gordura animal (pele de frango) e $13 \%$ de gelo. A porção cárnea variou quanto à proporção de CMS de frango em substituição à carne de frango desossada manualmente. Foram processadas 11 formulações distintas de mortadelas de frango, cuja porção cárnea foi constituída de 100\% (F100), 90\% (F90), 80\% (F80), 70\% (F70), 60\% (F60), 50\% (F50), 40\% (F40), $30 \%$ (F30), 20\% (F20), 10\% (F10) e 0\% (F0) de CMS. Após o processamento, foram obtidas mortadelas sem olhadura, de $63 \mathrm{~mm}$ de diâmetro e com peso unitário de $500 \pm 50$ gramas, as quais foram mantidas à temperatura de $5^{\circ} \mathrm{C}$. Antes de serem avaliadas sensorialmente, procedeu-se à avaliação da qualidade microbiológica das mortadelas segundo os padrões vigentes (Brasil, 2001).

O conteúdo de lipídeos das formulações de mortadela foi determinado pelo método intermitente de Soxhlet modificado (AOAC,1984), o de proteína pelo método Kjeldahl (AOAC,1997). A determinação da umidade foi realizada em estufa à temperatura de $105^{\circ} \mathrm{C}$ (Brasil, 1981) e o resíduo mineral fixo em mufla à temperatura de $500-550^{\circ} \mathrm{C}$ (AOAC, 1997). A atividade de água foi determinada em aparelho AquaLab CX-2, com as amostras à temperatura constante de $15^{\circ} \mathrm{C}$. As análises para determinação da composição centesimal e da atividade de água foram procedidas em quatro replicatas por formulação de mortadela.

Para as análises sensoriais, sob condições laboratoriais, as mortadelas foram cortadas em fatias de $3 \mathrm{~mm}$ de espessura e servidas à temperatura de $10 \pm 2^{\circ} \mathrm{C}$, sendo apresentadas aos julgadores de forma monádica, aleatorizadas e codificadas com números aleatórios de três dígitos. As fatias de mortadelas foram servidas acompanhadas de água e biscoito cracker, empregados para limpeza bucal entre as degustações.

O recrutamento dos julgadores foi realizado com o auxílio de questionário distribuído a 50 indivíduos. $\mathrm{Na}$ etapa de pré-seleção, utilizou-se uma série de seis repetições de teste triangular por julgador (Chaves, 1993), com o próprio produto (F10 e F30). Dos 20 candidatos aprovados no recrutamento, 12 julgadores potenciais foram classificados para a etapa de treinamento. Após diversas sessões de treinamento, foi pro- duzida a ficha de avaliação das amostras, contendo 16 atributos sensoriais dispostos em escalas de $15 \mathrm{~cm}$, ancoradas a $1,5 \mathrm{~cm}$ das extremidades por termos de intensidade e a lista de referência e definição de cada um dos atributos, ambas obtidas por consenso. Para a seleção de julgadores empregou-se a prova de desempenho, segundo os procedimentos utilizados por huerta-Leidenz et al. (1996), em que foi simulada uma Análise Descritiva Quantitativa (ADQ) em seis mortadelas (F0, F20, F40, F60, F80, F100, as quais foram testadas, em quatro repetições, pelos 12 julgadores. Para avaliação do desempenho dos julgadores utilizou-se a metodologia descrita por Damásio e Costell (1991), a qual considerada a habilidade discriminada e a reprodutibilidade dos julgadores, a partir da análise de variância (ANOVA) sobre os resultados de cada julgador com cada atributo avaliado. Os candidatos que

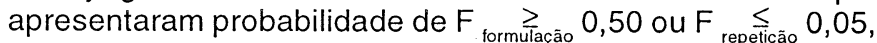
em um ou mais atributos, não foram considerados aptos a prosseguirem com os testes. Após esta etapa, foi obtida a equipe sensorial de oito julgadores treinados e selecionados, cinco mulheres e três homens, na faixa etária de 25 a 40 anos, a qual procedeu à análise descritiva quantitativa das 11 formulações de mortadela.

Para avaliar o efeito da adição de CMS, sobre as características sensoriais das mortadelas, foram ajustadas equações de regressão $(p<0,05)$ sobre os escores obtidos em cada atributo e, em seguida, testou-se o desvio da regressão $(p>0,15)$. Todas as análises estatísticas foram realizadas por procedimentos do pacote estatístico SAS (SAS Institute Inc., 1999), licenciado para a Universidade Federal de Viçosa.

\section{Resultado e discussão}

No presente trabalho, as formulações testadas buscaram otimizar o emprego da CMS e a qualidade sensorial do produto final. Nesse sentido, a variação do percentual de CMS foi acompanhada de variações nos percentuais de proteína de soja e de gordura animal. Este fato, provavelmente, concorreu para a baixa variaçãc da composição centesimal das mortadelas estudadas (Quadro 1).

A Carne Mecanicamente Separada (CMS) de frango, empregada para a produção das mortadelas testadas, continha 22,5 $\pm 0,31 \%$ de lipídeos, $69,48 \pm 0,99 \%$ de umidade, $11,6 \pm 0,39 \%$ de proteína e $0,93 \pm 0,02 \%$ de cinzas.

A avaliação da qualidade microbiológica das 11 formulações de mortadela demonstrou que todas as amostras se apresentaram dentro dos padrões microbiológicos vigentes (Brasil, 2001).

$\mathrm{Na}$ literatura consultada, não foi encontrado trabalho passível de comparação com os resultados apresentados no Quadro 1. A Instrução Normativa $n^{0} 21$ (Brasil, 1999), a qual estabelece o Regulamento Técnico de Identidade e Qualidade para Mortadela, prevê para o produto Mortadela de Carne de Ave a adição máxima de $40 \%$ de CMS e no produto final o limite máximo de umidade em $65 \%$, o limite mínimo de proteína em $12 \%$ e o limite máximo de gordura em $30 \%$. Comparando-se os limites estabelecidos para o produto em discussão com os valores dispostos no Qu:adro 1, observa-se que os produtos testados se apresentaram dentro dos limites estabelecidos para os teores de gordura e proteína. As formulações $\mathrm{F} 60, \mathrm{~F} 50, \mathrm{~F} 20, \mathrm{~F} 10$ e F0 apresentaram percentuais de umidade um pouco acima do previsto, variando entre 63 e $67 \%$. 
Quadro 1 - Valores médios da composição centesimal e da atividade de água de mortadelas formuladas com diferentes proporções de CMS de frango (100 a 0\%), em substituição à carne de frango desossada manualmente.

\begin{tabular}{|l|c|c|c|c|c|}
\hline \multicolumn{1}{|c|}{ Amostra } & $\%$ Lipídeos $^{*}$ & \% Proteína & \% Umidade* & \% Cinzas* & Atividade água \\
\hline F100 & $17,06 \pm 0,29$ & $13,14 \pm 0,39$ & $64,33 \pm 0,42$ & $3,14 \pm 0,01$ & $0,97 \pm 0,0006$ \\
\hline F90 & $17,67 \pm 0,05$ & $13,64 \pm 0,75$ & $62,43 \pm 0,30$ & $2,86 \pm 0,01$ & $0,98 \pm 0,001$ \\
\hline F80 & $17,05 \pm 0,07$ & $13,94 \pm 0,36$ & $62,71 \pm 0,67$ & $2,90 \pm 0,04$ & $0,98 \pm 0,001$ \\
\hline F70 & $15,56 \pm 0,19$ & $14,13 \pm 0,22$ & $62,37 \pm 0,33$ & $3,22 \pm 0,11$ & $0,99 \pm 0,005$ \\
\hline F60 & $15,88 \pm 0,31$ & $13,03 \pm 0,61$ & $65,70 \pm 2,16$ & $3,28 \pm 0,30$ & $0,99 \pm 0,002$ \\
\hline F50 & $15,08 \pm 0,07$ & $13,88 \pm 0,16$ & $65,71 \pm 0,52$ & $3,10 \pm 0,03$ & $0,99 \pm 0,001$ \\
\hline F40 & $15,44 \pm 0,06$ & $12,52 \pm 0,22$ & $63,42 \pm 0,61$ & $3,00 \pm 0,02$ & $0,99 \pm 0,0006$ \\
\hline F30 & $15,36 \pm 0,25$ & $12,58 \pm 0,63$ & $63,26 \pm 0,19$ & $2,96 \pm 0,05$ & $0,99 \pm 0,001$ \\
\hline F20 & $15,48 \pm 0,29$ & $12,05 \pm 0,15$ & $65,88 \pm 1,75$ & $3,07 \pm 0,08$ & $0,99 \pm 0,001$ \\
\hline F10 & $16,14 \pm 0,11$ & $12,07 \pm 0,38$ & $66,06 \pm 0,04$ & $3,00 \pm 0,04$ & $0,99 \pm 0,001$ \\
\hline F0 & $15,85 \pm 0,24$ & $11,68 \pm 0,07$ & $65,63 \pm 0,99$ & $3,07 \pm 0,02$ & $0,99 \pm 0,001$ \\
\hline
\end{tabular}

* Percentual médio obtido de análise em triplicata \pm desvio-padrão.

$\mathrm{F} 100$ a $\mathrm{FO}=$ mortadelas com porções cárneas variando em 100 a $0 \%$ de CMS.

As intensidades médias dos atributos de aroma, sabor, textura e aparência das mortadelas contendo 0,50 e $100 \%$ de CMS estão demonstradas nos perfis sensoriais (Figura 1). Estes sugerem variações de intensidade em decorrência da variação do conteúdo de CMS, destacando-se, os atributos cor, sabor de CMS e maciez. Os atributos sabor picante na massa e sabor picante residual não variaram em função do percentual de CMS. Este fato pode ser devido às mortadelas testadas não possuírem variação quanto aos percentuais de condimentos em suas formulações. O perfil sensorial também sugere que as variações nas intensidades do atributo sabor de soja foi nula ou muito baixa, indicando que a variação de 3 a $6 \%$ de proteína de soja, adicionada sob as formas texturizada e isolada às mortadelas, contribuiu de forma irrelevante na intensidade de percepção desse atributo.

No Quadro 2 estão apresentados os modelos de equação linear e quadrático que meIhor se ajustaram aos escores de intensidade dos atributos descritivos em função dos niveis de CMS das formulações testadas.
Quadro 2 - Modelos de equação de regressão de 16 atributos sensoriais (Y) em função dos níveis de CMS de frango $(x)$ adicionados em mortadelas e seus respectivos coeficientes de determinação $\left(R^{2}\right)$ e níveis de probabilidade $(p)$.

\begin{tabular}{|l|l|c|c|}
\hline Atributos de aroma & \multicolumn{1}{|c|}{ Modelo de regressão } & $R^{2}$ & prob>F \\
\hline 1. Soja & $Y=5,68-0,12 \cdot x$ & 0,70 & 0,0122 \\
2. CMS & $Y=9,35-0,89 \cdot x$ & 0,91 & 0,0001 \\
3. Característico de mortadela & $Y=8,83-0,33 \cdot x$ & 0,86 & 0,0001 \\
\hline Atributos de sabor & & & \\
& & & \\
\hline 4. Soja & $Y=4,48+0,06 \cdot x$ & 0,50 & 0,1257 \\
5. CMS & $Y=9,94-0,07 \cdot x$ & 0,92 & 0,0001 \\
6. Picante na massa & $Y=6,19-0,03 \cdot x$ & 0,07 & 0,6044 \\
7. Característico de mortadela & $Y=9,23-0,34 \cdot x$ & 0,88 & 0,0001 \\
8. Picante residual & $Y=6,63-0,08 \cdot x$ & 0,32 & 0,2271 \\
\hline Atributo de textura & & & \\
\hline 9. Elasticidade & $Y=2,05+1,51 \cdot x-0,078 \cdot x^{2}$ & 0,96 & 0,0001 \\
10. Maciez & $Y=13,32-1,59 \cdot x+0,077 \cdot x^{2}$ & 0,96 & 0,0001 \\
11. Coesividade & $Y=1,50+1,37 \cdot x-0,064 \cdot x^{2}$ & 0,95 & 0,0001 \\
12. Arenosidade na massa & $Y=6,35-0,43 \cdot x$ & 0,94 & 0,0001 \\
13. Oleosidade da massa & $Y=6,26-0,22 \cdot x$ & 0,96 & 0,0001 \\
14. Arenosidade residual & $Y=5,23-0,37 \cdot x$ & 0,95 & 0,0001 \\
15. Filme de óleo residual boca & $Y=6,07-0,21 \cdot x$ & 0,97 & 0,0001 \\
\hline \multicolumn{1}{|c|}{ Atributo de aparência } & & & \\
\hline 16. Cor & $Y=13,05-0,84 \cdot x$ & 0,98 & 0,0001 \\
\hline
\end{tabular}

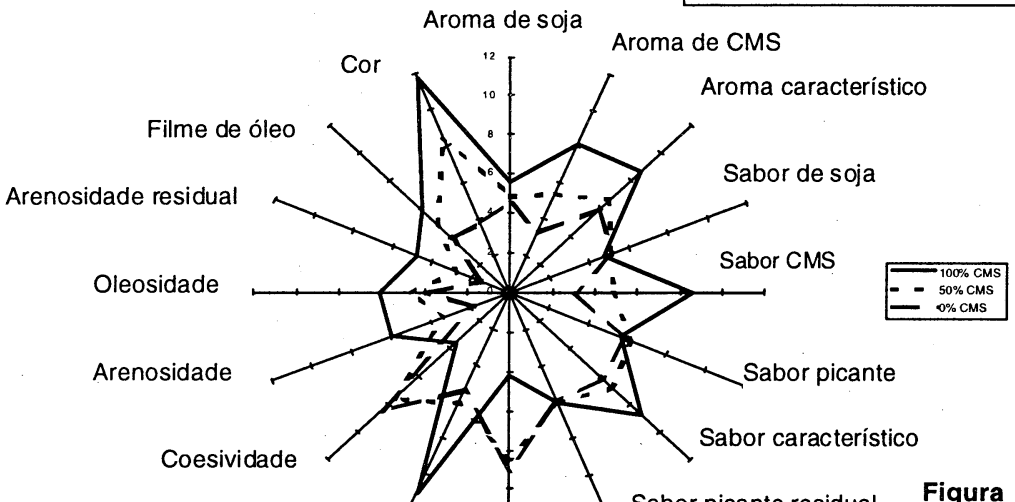

Sabor picante residual

Maciez

Elasticidade
Observa-se, no Quadro 2, que ocorreu correlação linear significativa em diversos atributos e, ainda, correlações quadráticas significativas dos atributos de textura: elasticidade, maciez e coesividade em função do conteúdo de CMS das mortadelas estudadas. Corroborando os resultados da Figura 1, baixos valores de $R$ e baixos níveis de probabilidade $(p)$ foram obtidos nas equações de regressão dos atributos sabor picante na massa, sabor picante residual e sabor de soja.

As variações de intensidade dos atributos sensoriais em função do percentual de CMS, os quais se apresentaram significativos na análise de regressão, estão demonstradas nas Figuras 2 a 6 . 

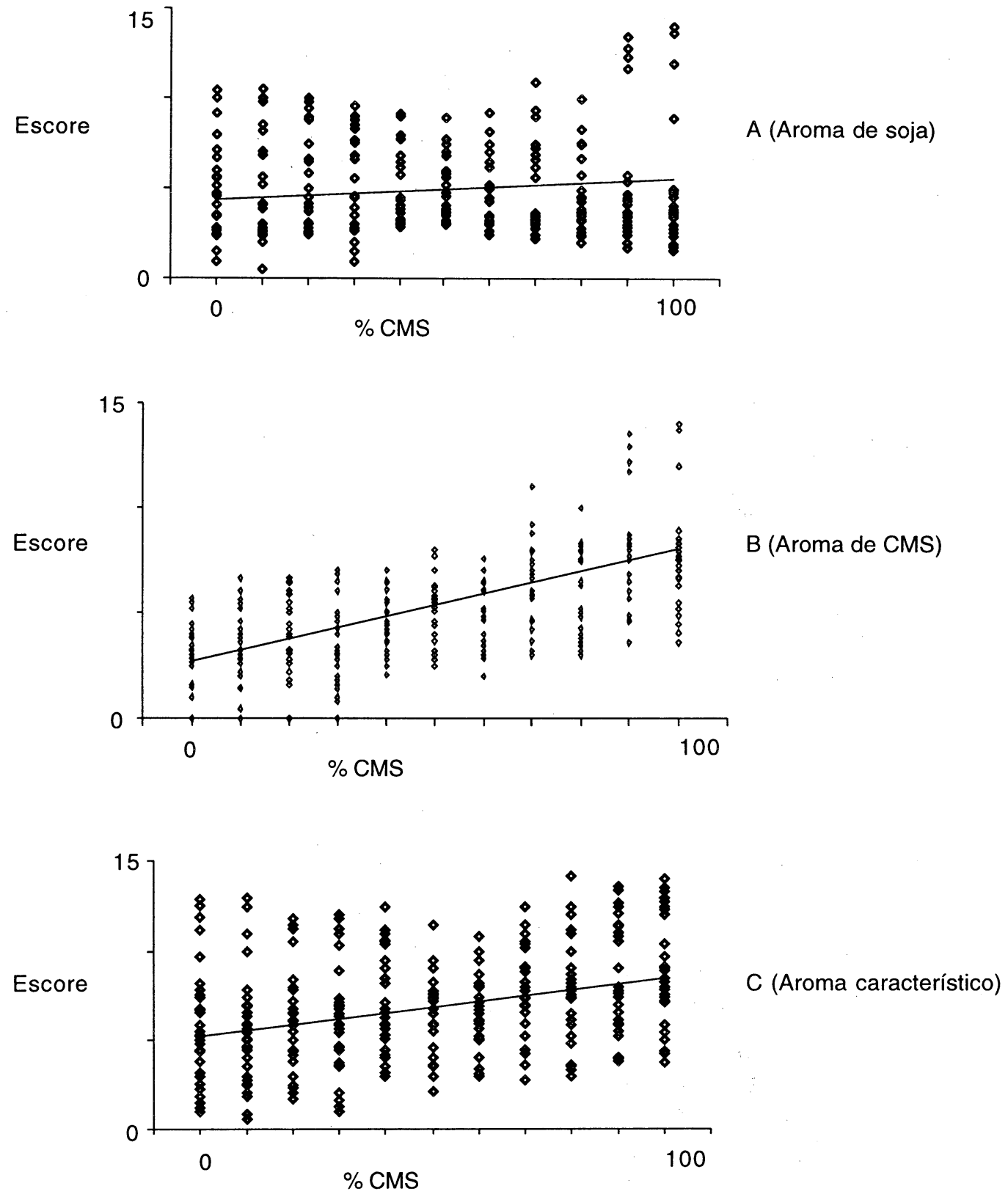

Figura 2 - Variações dos escores sensoriais dos atributos de Aroma: de soja (A), de CMS (B) e característico de mortadela (C) em mortadelas formuladas com diferentes percentuais de CMS de frango (0 a $100 \%$ da porção cárnea). 


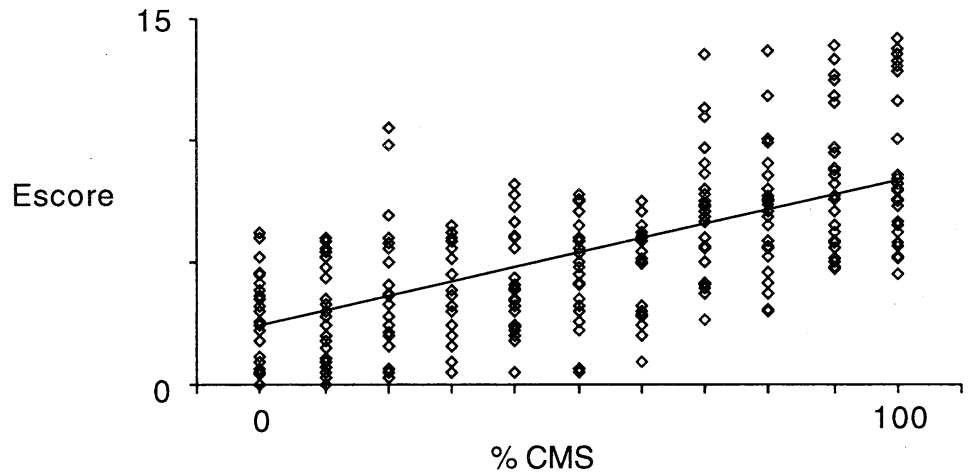

A (Sabor de CMS)

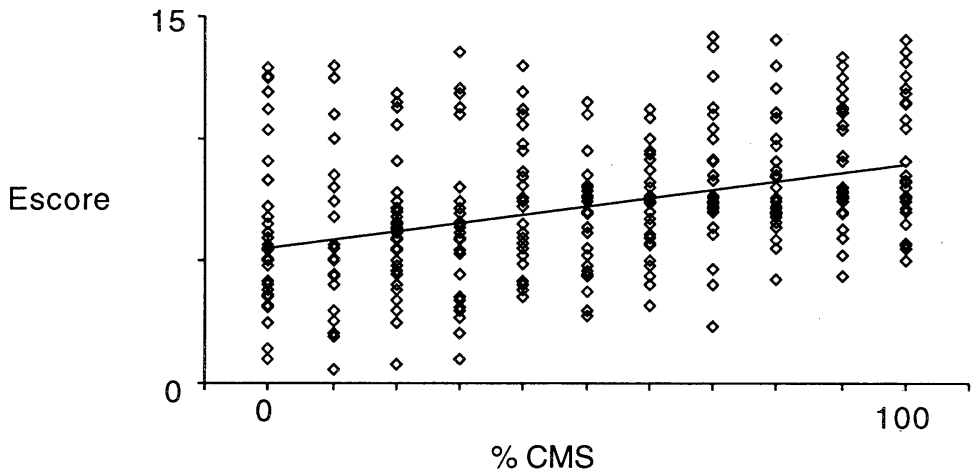

B (Sabor característico)

Figura 3 - Variações dos escores sensoriais dos atributos de Sabor: de CMS (A) e característico de mortadela (B) em mortadelas formuladas com diferentes percentuais de CMS de frango (0 a 100\% da porção cárnea). 

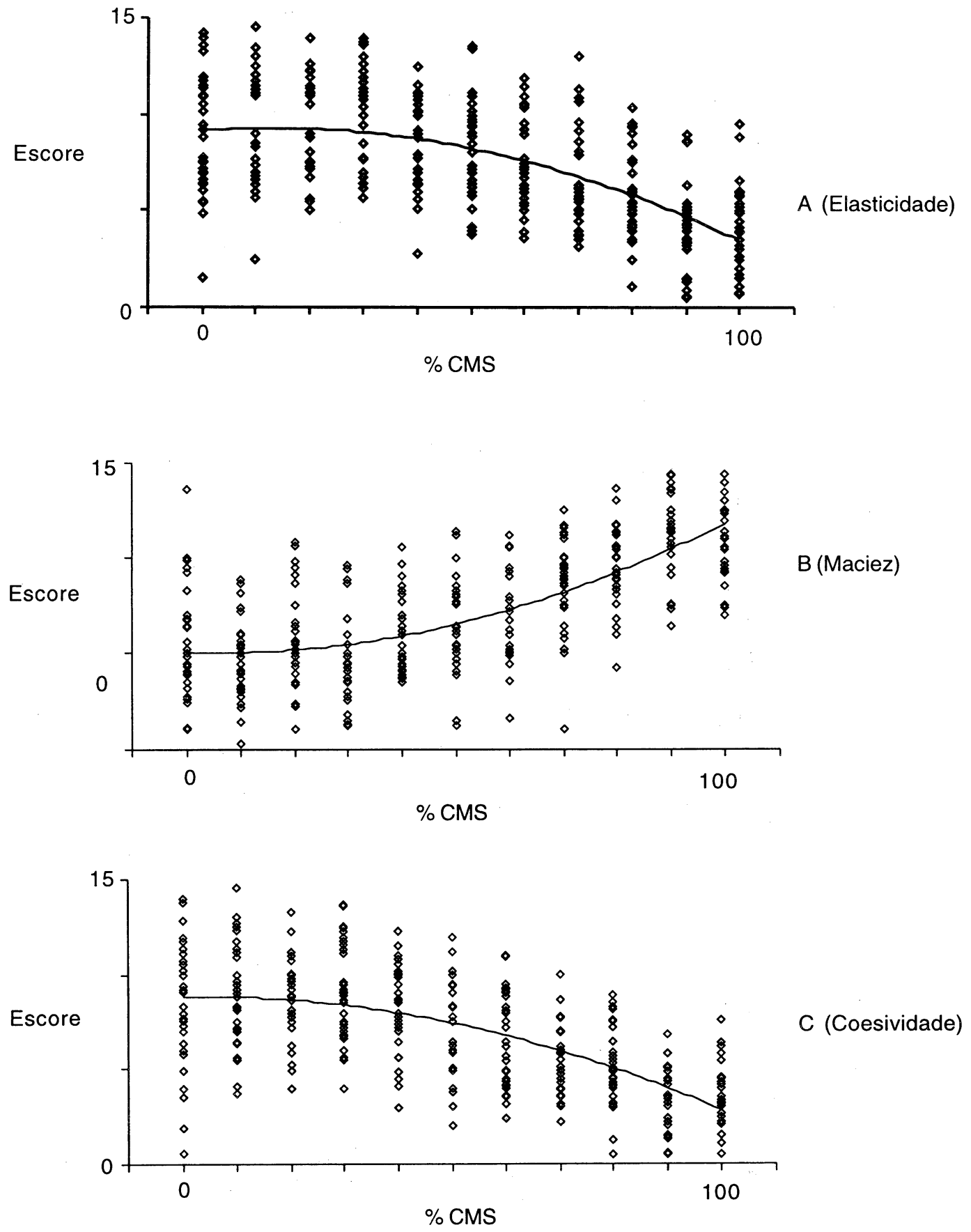

Figura 4 - Variações dos escores sensoriais de atributos de Textura: elasticidade (A), maciez (B) e coesividade (C) em mortadelas produzidas com diferentes percentuais de CMS de frango ( 0 a $100 \%$ da porção cárnea). 

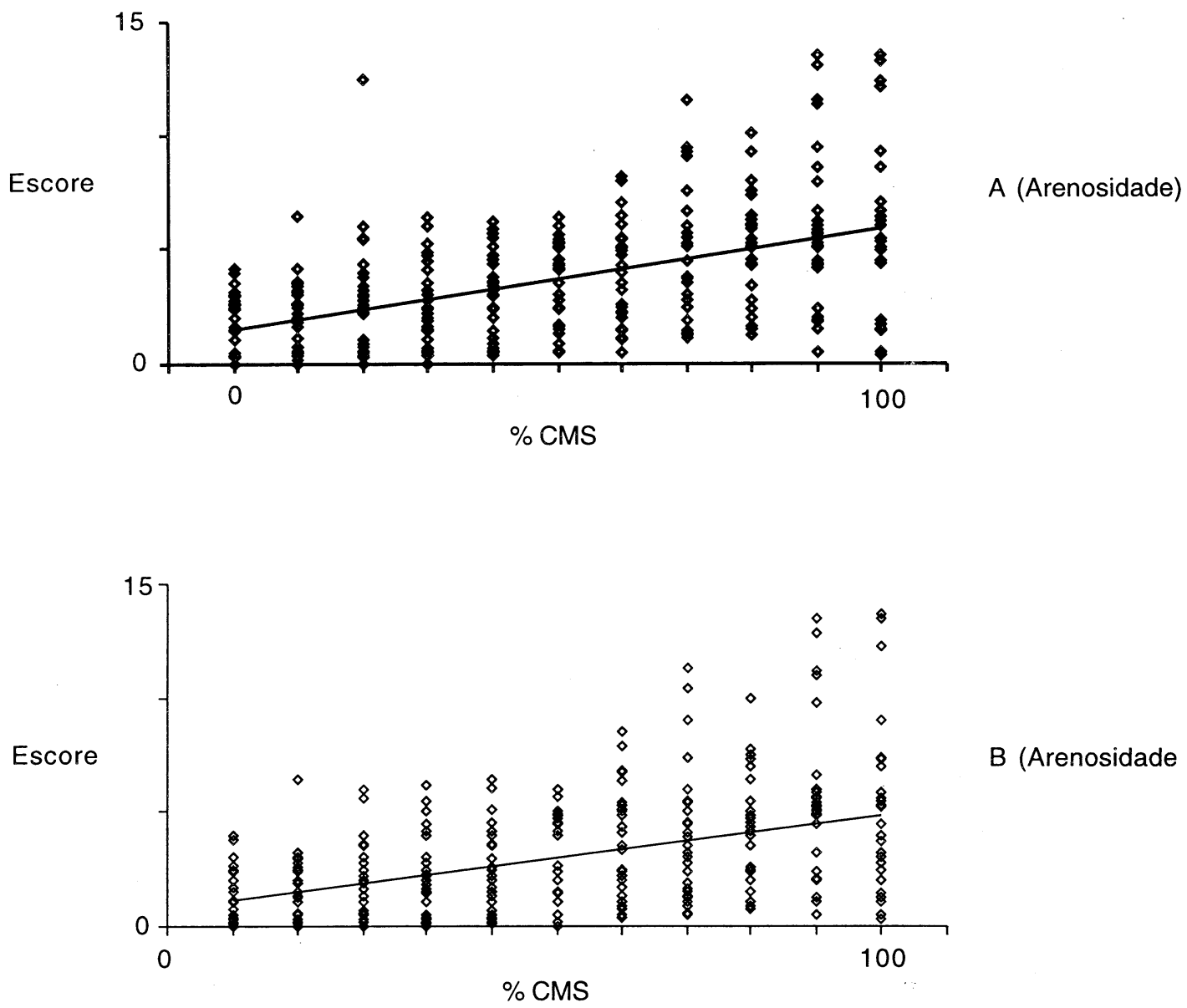

Figura 5 - Variações dos escores sensoriais dos atributos de Textura: arenosidade (A) e arenosidade residual (B) em mortadelas formuladas com diferentes percentuais de CMS de frango (0 a 100\% da porção cárnea). 


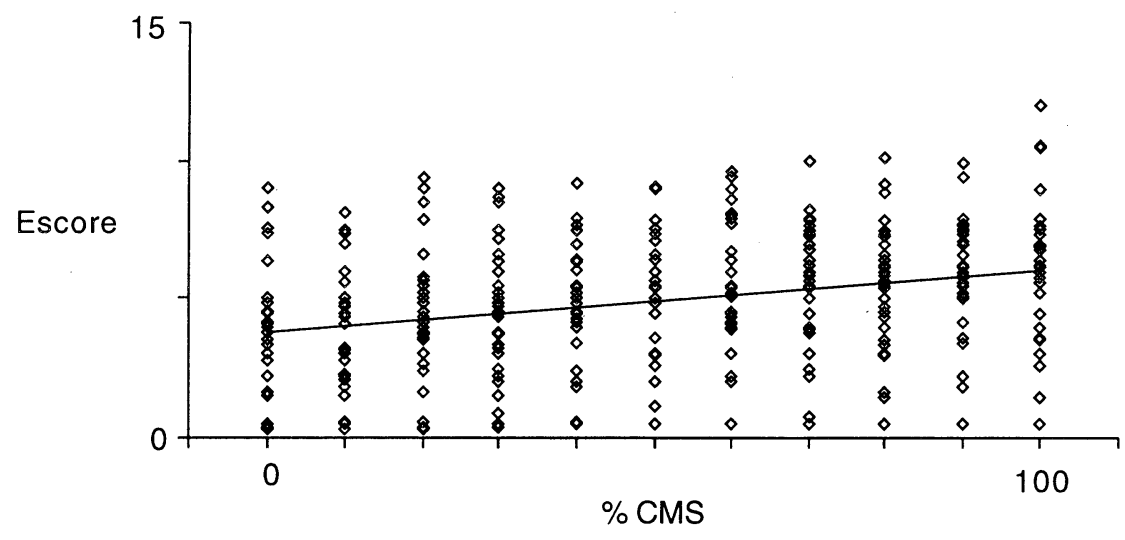

A (Oleosidade)

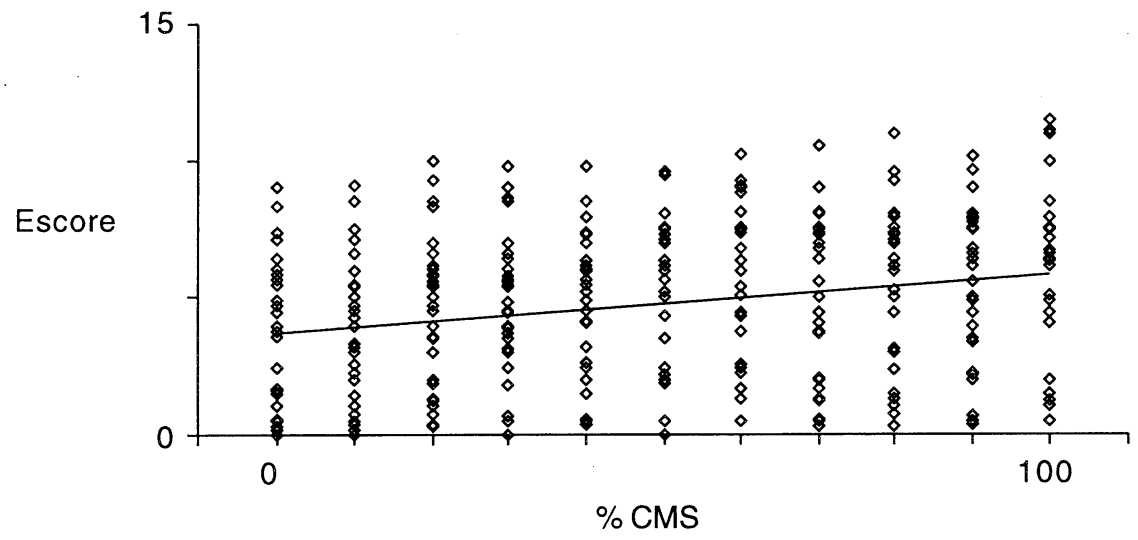

B (Filme de óleo)

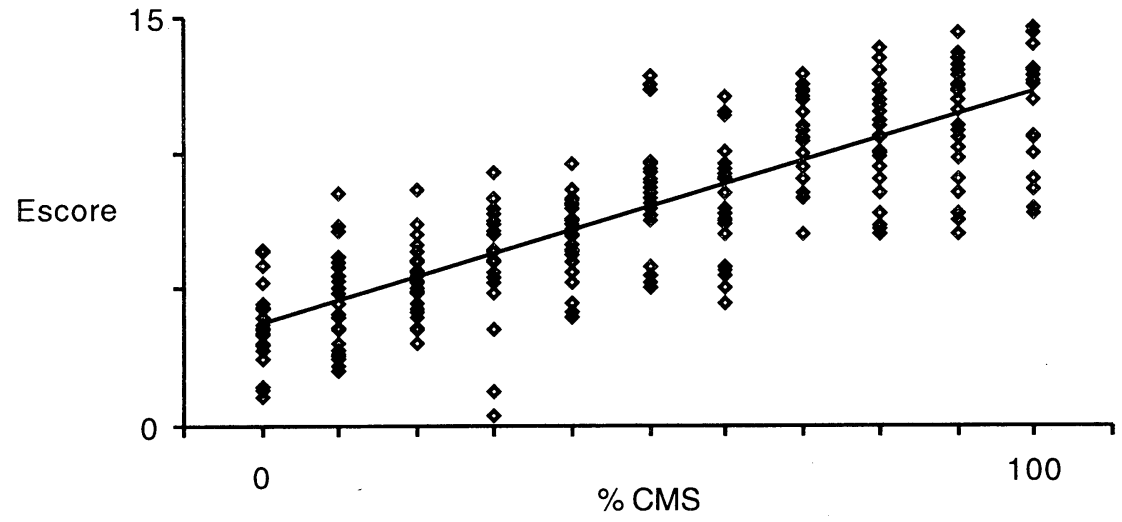

Figura 6 - Variações dos escores sensoriais de atributos de Textura: oleosidade na massa (A), filme de óleo residual na boca (B) e de atributo de Aparência: cor (C) em mortadelas produzidas com diferentes percentuais de CMS de frango (0 a $100 \%$ da porção cárnea). 
Observa-se, na Figura 2A, que a intensidade de percepção do atributo aroma de soja aumentou em função do percentual de CMS, podendo ser explicado pelo fato de que o aumento do percentual de CMS foi acompanhado do aumento do percentual de proteína de soja adicionado às formulações. Corroborando os resultados apresentados na Figura 1, o aumento do percentual de CMS intensificou a percepção dos atributos aroma e sabor de CMS, definidos como aroma e sabor cárneo mais ativos que os percebidos na carne de frango desossada manualmente, respectivamente (Figuras $2 \mathrm{~B}$ e $3 \mathrm{~A}$ ).

Nas Figuras $2 \mathrm{C}$ e $3 \mathrm{~B}$, observa-se que a adição de CMS intensificou a percepção dos atributos aroma e sabor característicos, definidos como sendo aqueles percebidos nas mortadelas tradicionais, as quais são produzidas com carne de diversos animais de açougue. Tais resultados sugerem que o emprego da CMS de frango intensificou o aroma e sabor cárneo das mortadelas testadas, aproximando-as das mortadelas tradicionais.

$\mathrm{Na}$ Figura 4, observa-se que o aumento do percentual de CMS, de modo geral, intensificou a maciez e reduziu a elasticidade e coesividade das formulações estudadas, concordando com os resultados obtidos por McMillin et al. (1980) e Meullenet et al. (1994) e corroborando as observações de Shand et al. (1990) e Froning (1981), de que a CMS produz ligações protéicas mais débeis nas emulsões cárneas. Os resultados sensoriais demonstraram, ainda, que a debilidade das ligações protéicas da CMS reduziu o grau com que as mortadelas retornavam à forma original (elasticidade) e a coesão entre suas partículas (coesividade).

Derivando-se a equação de regressão do atributo elasticidade apresentada no Quadro 6, observou-se o ponto de máxima elasticidade no percentual de $9,44 \%$ de CMS. Da mesma forma, observou-se o ponto de mínima maciez no percentual de $9,94 \%$ de CMS e de máxima coesividade em $11,42 \%$ de CMS.

$\mathrm{Na}$ Figura 5, observa-se o aumento de intensidade dos atributos arenosidade na massa e arenosidade residual em função do maior percentual de CMS adicionado ao produto, o que está em consonância com as observações de Dawson e Gartner (1983). Este fato é particularmente observado em CMS de frango, devido ao abate de animais jovens, em média com 45 dias de idade, cujos ossos não estão completamente calcificados.

\section{Referências}

AOAC, Association of Official Analytical Chemists, Official Methods of Analysis. Arlington, D.C., USA, 1984.

AOAC, Association of Official Analytical Chemists, Official Methods of Analysis. Washington D.C., USA, 1997.

BRASIL, Ministério da Agricultura. Agência Nacional de Vigilância Sanitária. Resolução n² 12 do Diário Oficial da União, n 7-E, seção 1, p. 45-53 em 10 de Janeiro de 2001

BRASIL, Ministério da Agricultura. Secretaria de Defesa Agropecuária e do Abastecimento. Portaria no 574, de 8/12/1998. Instrução Normativa no 21 de 21/7/1999.

BRASIL, Ministério da Agricultura. Secretaria Nacional de Defesa Agropecuária. Laboratório Nacional de Referência Animal (LANARA). Métodos analíticos oficiais para controle de produtos de origem animal e seus ingredientes. II. Métodos físico-químicos. Aprovado pela Portaria $n^{0} 001$ de 7/10/81. Brasília, 123p.1981.
Mesmo tendo ocorrido baixa variação no conteúdo de lipídeos nas amostras estudadas (Quadro 1), a Figura 6(A/B) sugere diferenças perceptíveis nos atributos oleosidade e filme de óleo residual. Comc o processo de desossa mecânica incorpora fosfolipídeos provenientes da medula óssea (Froning, 1981 e Raphaelides et al.,1998), a variação do percentual de pele adicionado às mortadelas pode não ter sido suficiente para compensar o maior conteúdo de lipídeos da CMS (24\%), contra $3,2 \%$ encontrados no músculo de coxa e sobrecoxa de frango por Pikul e Kummerow (1990). Alia-se, ainda, o fato da pele de frango possuir alto conteúdo de colágeno. Neste sentido, o aumento do conteúdo de pele nas formulações contendo menores percentuais de CMS, pode não ter sido suficiente para repor o conteúdo de lipídeos dessas mortadelas.

Observa-se, na Figura 6(C), que a substituição da carne de frango desossada manualmente pela CMS de frango aumentou a intensidade do atributo cor, o qual passou de salmão pálido a corado. Com o aumento do percentual de CMS, as mortadelas de frango se aproximaram da coloração de mortadela tradicional, obtida com carne de diversos animais de açougue. Este fato, segundo Froning (1981) e Raphaelides et al. (1998), se deve à incorporação de hemopigmentos provenientes da medula óssea durante o processo de desossa mecânica.

\section{Conclusões}

A composição centesimal de mortadelas formuladas com porção cárnea variando entre 0 e $100 \%$ de CMS de frango, em substituição à carne de coxa e sobrecoxa desossada manualmente, em geral, se apresentou dentro dos padrões de identidade e qualidade estabelecido para o produto.

Sensorialmente, a adição de CMS aumentou a intensidade de percepção dos atributos, aroma e sabor de soja, arenosidade na massa, arenosidade residual, oleosidade na massa e filme de óleo residual na boca. De modo geral, o aumento do percentual de CMS foi acompanhado da redução da elasticidade, da coesividade e da dureza das mortadelas. Nas mortadelas de frango estudadas, ficou claramente demonstrado que a adição de CMS contribuiu para acentuar a cor, o aroma e sabor de CMS, e o aroma e sabor característi$\mathrm{co}$, aproximando-as das mortadelas tradicionais, produzidas com carne de diversos animais de açougue.

CHAVES, J.B.P. Métodos de diferença em avaliação sensorial de alimentos e bebidas. Viçosa: Universidade Federal de Viçosa. Imprensa Universitária, 1993.

DAMÁSIO, M.H., COSTELL, E. Análisis sensorial descriptivo: generación de descriptores y selección de catadores. Ver. Agroquim. Tecnol Aliment., v 31, n. 2, p 165-178, 1991.

DAWSON, L.E., GARTNER, R. Lipid oxidation in mechanically deboned poultry. Food Technology, v. 37, n. 7, p. 112-117, 1983.

FIELD, R.A .Mechanically deboned meat. Proceeding of the meat industry research conference, n. 21/22, p. 35-42, 1974.

FRONING, G.W. Mechanical deboning of poultry and fish. Advances in Food Research, v 27, p 109-147, 1981.

FRONING, G.W. Mechanically-deboned poultry meat. Food Technology, v. 35, n. 9/12, p 50-63, 1976

HAMM, D., YOUNG, L.L. Further studies on the composition of commercially prepared mechanically deboned poultry meat. Poultry Science, v. 62, p. 1810-1815, 1983. 
HUERTA-LEIDENZ, N., JEREZ-TIMAURE, N., MORÓN-FUENMAYOR, O., URDANETA, E.R., CARO, R. Experiencias en el entrenamiento de un panel de degustación de carne vacuna a nivel de um matadero frigorífico industrial venezolano. Archivos Latinoamericanos de Nutrición, v. 46, n. 1, p. 47-53, 1996.

LEE, Y.B., HARGUS, G.L., KIRKPATRICK, J.A , BERNER, D.L. , FORSYTHE, R.H. Mechanism of lipid oxidation in mechanically deboned chicken meat. Journal of Food Science, v. 40, p. 964-967, 1975.

MEULLENET, J.F., CHANG, H.C., CARPENTER, J.A., RESSURRECCION, A.V.A. Textural properties of chicken Frankfurters with added collagen fibers. Journal of Food Science, v. 59, n. 4, p. 729-733, 1994.

McMAHON, E.C., DAWSON, L.E. Effects of sat and phosphates on some functional characteristics of hand and mechanically deboned turkey meat. Poultry Science, v. 55, p. 573-578, 1976.

MCMILLIN, K.W., SEBRANEK, J.C., RUST, R.E., TOPEL, D.G. Chemical and physical characteristics of frankfurters prepared with mechanically processed pork product. Journal of Food Science, v. 45, n. 6, p. 14551459, 1462, 1980.
MOERCK, K.E., BALL, JrH.R. Lipid autoxidation in mechanically deboned chicken meat. Journal. of Food Science, v. 39, p. 876-879, 1974.

PIKUL, J., KUMMEROW, F.A. Lipid oxidation in chicken muscles and skin after roasting and refrigerated storage of main broiler parts. Joumal of Food Science, v. 44, n. 1, p. 30-37, 1990.

RAPHAELIDES, S.N., GRIGOROPOULOU, S., PETRIDIS, D. Quality attributes of pariza salami as influenced by the addition of mechanically deboned chicken meat. Food Quality and Preference, v. 9, n. 4, p. 237242, 1998.

SAS Institute. SAS ${ }^{R}$ User's Guide. 6.04 Edition. SAS Institute Inc., Cary, NC.1999.

SCHNELL, P.G., VADEHRA, D.V., HOOD, L.R., BAKER, R.C. Ultrastructure of mechanically deboned poultry meat. Poultry Science, v. 53, p. 416-419, 1974.

SHAND, P.J., SCHIMIDT, G.R., MANDIGO, R.W., CLAUSS, J.R. New technology for low fat meat products. Reciprocal Meat Conference Proceedings. Mississippi, v. 43, p. 37-45, 1990. 